\title{
The effect of context on sensory accumulation involved in decision-making
}

\author{
Léa Caya-Bissonnette
}

\begin{abstract}
The underlying processes allowing for decision-making has been a question of interest for many neuroscientists. The lateral intraparietal cortex, or LIP, was shown to accumulate context and sensory information to compute a decision variable. The following review will present the work of Kumano, Suda and Uka who studied the link between context and sensory information during decision-making. To do so, a monkey was trained to associate the color of a fixating dot to one of two tasks. The tasks consisted in either indicating the motion or the depth of the majority of the dots on a screen. The local field potential of the LIP neurons was recorded, and the researchers found a role of context during the stimulus presentation in regards to decision formation. The results have important implication for mental disorders involving malfunction in decision processes.
\end{abstract}

Keywords: Decision-making; Task-switching; Sensory accumulation

\section{Résumé}

Les processus sous-jacents permettant la prise de décision ont été une question d'intérêt pour de nombreux neuroscientifiques. On a montré que le cortex intrapariétal latéral, ou IPL, accumulait des informations contextuelles et sensorielles pour calculer une variable de décision. La revue suivante présentera le travail de Kumano, Suda et Uka qui ont étudié le lien entre le contexte et l'information sensorielle lors de la prise de décision. Pour ce faire, on a entraîné un singe afin qu'il puisse associer la couleur d'un point de fixation à l'une de deux tâches. Les tâches consistaient à indiquer le mouvement ou la profondeur de la majorité des points sur un écran. On a enregistré le potentiel de champ local des neurones IPL. D'après ceci, les chercheurs ont découvert un rôle de contexte lors de la présentation du stimulus en ce qui concerne la formation de la décision. Les résultats ont une implication importante pour certains troubles mentaux concernant un dysfonctionnement dans les processus de prise de décision.

Mots Clés: La prise de décision; Flexibilité mentale; Flexibilité

Understanding the neural basis behind decision formation in task switching performance is crucial to mapping out the extremely complex and dynamic maze that is the human brain. The impact of this understanding on the scientific community and the medical field would be significant, as decision-making is linked to some neurological diseases such as schizophrenia (1). Specific tasks are represented through sensory, contextual and motor components in the prefrontal neurons (2). However, these neurons do not seem to be independently involved in the formation of decisions. As Gold and Shadlen demonstrated, an LIP neuron also accumulates context and sensory information to compute a decision variable (3).

Correspondence: Icaya082@uottawa.ca

Department of Biology, University of Ottawa, 30 Marie Curie, K1N 6N5,

Ottawa, Canada
Tasks involving both contextual and sensory components, such as the game "Where is Waldo?" are prevalent. The game requires the player to find a character hidden in a complex environment. Contextual components such as finding Waldo, a character wearing a striped red and white t-shirt, help the player to perform the task efficiently. Interestingly, few have looked at the potential interdependence of these two variables. Therefore, this raise an important question: would context influence the intake of sensory evidence?

The researchers Kumano, Suda, and Uka found an answer to this question by investigating if and how decision formation changes depending on context (4). To do so, they measured the extracellular activity of a single LIP neuron of two monkeys during task switching. The monkeys were presented with a multitude of red dots with dif- 
ferent depths and directions of motion. They were then trained to indicate either the depth or the direction of the majority of the dots based on the color of a fixating point. The change in color of the fixating point represents the variation in context. In other words, even if presented with similar sensory inputs, the relevant stimulus to the task varied according to contextual components. The two tasks were randomly interwoven and were chosen for their previously established sensory representation $(5,6)$. To respond, the monkeys were required to make an eye movement, a saccade, to one of two targets. An upper target was associated with an upward motion or farther disparity of the red dot stimuli, and a lower target, which was diametrically opposite to the upper one, was associated with a downward motion or nearer disparity. Up to 128 stimuli conditions with different motion coherence and binocular correlations were recorded with their average time of reaction. To find the receptive field of the neuron, Kumano, Suda, and Uka recorded the firing rate of the LIP neuron for the upper target choice by using a memory-guided saccade task.

The researchers built a psychometric function which plotted strength of the relevant stimulus against accuracy of their choice and a chronometric function to plot strength of the relevant stimulus against time reaction. Both functions suggest that the higher the strength of the irrelevant stimulus, the less accurate and the longer the monkeys took to make a choice. More importantly, the firing rate of the relevant stimulus to the task had a shorter time of build-up than the irrelevant stimulus after stimuli onset, but both reached a common level right before saccade, meaning that the accumulation of the relevant stimulus is preferential to the irrelevant stimulus. However, the authors did not calculate the time differences between build-up onset of the relevant and irrelevant stimuli, giving no quantitative data of this preferentiality. To investigate whether this sensory accumulation occurs at the level of a single neuron, researchers Kumano, Suda, and Uka measured the sensitivity of an LIP neuron to motion coherence and to binocular correlation during both tasks (4). They observed a change in build-up rates related to the change in the strength of the relevant stimulus and found that most single neurons will fire for both stimuli when they are relevant. Then, they compared the sensitivity of the relevant stimulus to the irrelevant stimulus and concluded that the accumulation of the relevant feature was more prominent than the irrelevant one. This finding suggests that context does influence the preferential accumulation of a single LIP neuron. Nonetheless, some questions remain. Since the LIP neuron computes a decision variable but is not related to task error, task selection is at least partly computed elsewhere in the brain. Using electrophysiology techniques, future studies could potentially reveal the pathway leading to decision of task selection. In addition, neurological diseases such as schizophrenia underlie cognitive impairment of task switching performance, resulting in more latency and less accuracy of the saccade response (3). Evaluating the effect of context by measuring and comparing the sensitivity to relevant and irrelevant stimuli of a schizophrenic individual to a non-schizophrenic subject would be an interesting follow-up and could result in a major breakthrough in the field of neuropsychiatric diseases. To conclude, the preferential accumulation of LIP neurons for relevant information serves as a crucial step towards uncovering the underlying processes of decisionmaking.

Competing interests

The author declares that she has no competing interests.

References

1. C. Greenzang, D. S. Manoach, D. C. Goff, J. J. S. Barton, Exp Brain Res 181, 493 (2007).

2. M. Siegel, T. J. Buschman, E. K. Miller, Science 348, 1352 (2015).

3. J. I. Gold, M. N. Shadlen, Annu. Rev. Neurosci. 30, 535 (2007).

4. H. Kumano, Y. Suda, T. Uka, Journal of Neuroscience 36, 12192 (2016).

5. T. Uka, G. C. DeAngelis, J. Neurosci. 23, 3515 (2003).

6. T. Uka, G. C. DeAngelis, Neuron 42, 297 (2004). 